\title{
Obstacles Facing Developing Countries in Power System Planning
}

\author{
Abdullah M. Al-Shaalan \\ Electrical Engineering Department College of Engineering King Saud University, Riyadh, Saudi Arabia \\ Email: Shaalan123@gmail.com
}

Received October 2014

\begin{abstract}
The problem of power system planning, due to its complexity and dimensionality, is one of the most challenging problems facing the electric power industry in developing as well as developed countries. In planning phase, two of the most important decision-making parameters are the reliability and costs. The latter includes both system investment costs and outages costs. In this paper, these parameters are described and the interrelation between them is evaluated. Some previous approaches and developed techniques will he applied to a particular planning problem in a developing country and some aspects having a significant impact on the decision making process in the planning phase will be considered.
\end{abstract}

\section{Keywords}

Power System Planning, Developing Countries, Reliability Evaluation, System Cost, Outages Cost, Systems Interconnection, Load Uncertainty

\section{Introduction}

The main issue regarding power system planning in developing countries is to establish basic principles and criteria to serve as a framework within which the process of planning may proceed. The framework of power system planning should be flexible, with the broad objectives of finding a plan (or plans) which guarantee a desired degree of continuous and least cost service. Good service or, in other words, acceptable reliability level of a power system usually requires the addition of more generating capacity to meet the ever increasing electrical demand [1] [2]. However, in many fast developing countries with vast, sparsely populated areas reliability-cost tradeoffs exist to satisfy the fast load growth either by investment in additional generating capacity for isolated systems or by building transmission lines to interconnect these systems in such a way as to transfer power between their load centers in case of emergencies and power shortages. Therefore, reliability and cost constraints are major considerations in power system planning process [3].

\section{Reliability Aspects in Power System Planning}

Reliability is one of the most important criteria which must be taken into consideration during all phases of power system planning process. Reliability criterion is required by the system planers and authorized manage- 
ments in the utilities to establisharget reliability levels and to consistently analyze and compare the future reliability levels with feasible alternative expansion plans. This need has resulted in the development of comprehensive reliability evaluation in power system planning modeling and techniques [4]-[8].

One reliability index, known as the Loss of Load Expectation (LOLE), is presently the most commonly adopted and used probabilistic criterion in power system generation expansion planning. This index computes the expected (long term average) number of days per year on which the available generating capacity is not sufficient to supply all the period peak load levels. The first step in evaluating this index is to define models that describe two situations of interest, namely, the capacity model and the load model. These two models are convolved together to produce the risk index known as the LOLE.

In order to derive the capacity model, each unit in the system is represented by a 2-state capacity model (i.e. available full capacity or unavailable capacity). Each state is then weighted by its probability of occurrence. This process creates the capacity model known as the "Capacity Outage Probability Table, COPT" which contains an array of capacity states and their associated probabilities of occurrences.

The other load model needed for the LOLE index evaluation is known as the "Load Duration Curve, LDC" which represents an arrangement of a typical period individual load levels in a descending order of magnitude (i.e. starting with the peak load level and ending with the minimum load level) as the one shown in Figure 1.

\subsection{Loss of Load Expectation (LOLE)}

The two models: COPT and LDC mentioned above are to be combined together in order to yield the required reliability risk index LOLE as can be exhibited in the following equation:

$$
\text { LOLE }=\sum_{i=1}^{n} t_{i} \cdot p\left(O_{i}\right) \quad(\text { days/year }) \quad(\text { outage }>\text { Reserve })
$$

where

$t_{i}$ : time duration of that sever outage $O_{i}$

$p\left(O_{i}\right)$ : probability of loss of load due to the $i^{\text {th }}$ severe outage of size $O_{i}$

$n$ : total number of severe outages ocurred during that period considered

\subsection{Expected Load Not Served ( $\epsilon$ LNS)}

In power system reliability evaluation, sometimes another reliability index beside the $L O L E$ is needed to know the magnitude of loads that have been lost due to severe outages (i.e. when the existing loads exceed the available system capacity). So, this index is known as the Expected Load Not Served ( $\epsilon$ LNS) and can be evaluated as in the following equation:

$$
\left.\epsilon \mathrm{LNS}=\sum_{i=1}^{n}(\mathrm{LNS})_{i} \cdot p\left(O_{i}\right) \mathrm{MW} / y \quad \text { (outage }>\text { Reserve }\right)
$$

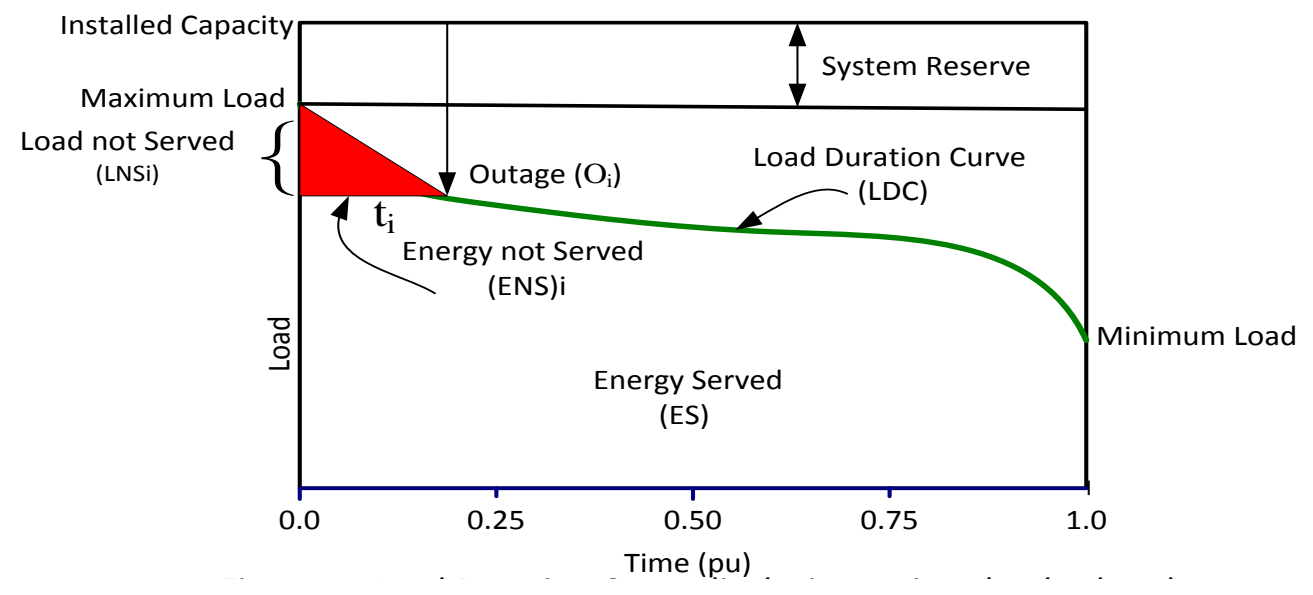

Figure 1. Load duration curve displaying various load-related variables. 


\subsection{Expected Energy Not Served ( $\epsilon$ ENS)}

Since the energy not served (ENS) ${ }_{i}$ caused by power outages reflects great damages and heavy losses to the entire consumers classes, so, another essential and most needed reliability index known as the Expected Energy not Served $(\epsilon$ ENS $)$ can be deduced as in the following equation:

$$
\epsilon \mathrm{ENS}=\sum_{i=1}^{n}(\mathrm{ENS})_{i} \cdot p\left(O_{i}\right) \mathrm{MWh} / y \quad(\text { outage }>\text { Reserve })
$$

where

$(\mathrm{ENS})_{i}=(\mathrm{LNS})_{i} \cdot t_{i}$ : The energy not served due to severe $i^{\text {th }}$ outage of size Oin time $t$

\section{Economic Aspects in Power System Planning}

There are several costs that are associated with power systems planning and can be manifested in the following sections

\subsection{Fixed Cost}

The fixed cost (FC) represents the cash flow at any stage of the planning horizon resulting from the costs of installing new generating units during the planning period. It depends on the current financial status of the utility, the type and size of generating units and the cost of time on money invested during the planning period. The total fixed costs $\left(\mathrm{FC}_{T}\right)$ for unit(s) being installed can be computed as:

$$
\mathrm{FC}_{T}=\sum_{t} \sum_{k}\left(\mathrm{CAP}_{k} \cdot \mathrm{CC}_{k} \cdot \mathrm{NU}_{k}\right)^{t}
$$

where

$\mathrm{CAP}_{k}$ : unit capacity added to the system of type $k$.

$\mathrm{CC}_{k}$ : capital cost of unit of type $k(\$ / \mathrm{kW})$.

$\mathrm{NU}_{k}$ : number of unit(s)added to the system of type $k$ at each interval of time $t$

$t$ : interval period of time considered in the planning horiozon, $t=1, \cdots, T$

\subsection{Variable Cost}

The variable cost, (VC), represents the cost of energy served by the system. It is affected by the load variation, the type and size of generating units and the number of hours of operation. Also these costs are related to the cost of operation and maintenance (fuel, scheduled maintenance, interim spare parts, repair, staffing, wages and miscellaneous expenses) and can be evaluated as:

$$
\mathrm{VC}_{T}=\sum_{t} \sum_{k}\left(\epsilon \mathrm{ES}_{k} \cdot \mathrm{ESC}_{k} \cdot \mathrm{NU}_{k}\right)^{t}
$$

where

$\epsilon \mathrm{ES}_{k}$ : expected energy served by unit of type $k$

ESC $_{k}$ : energy served cost of unit of type $k(\$ / \mathrm{kWh})$

The total system costs $\left(\mathrm{SC}_{T}\right)$ for the entire expansion plan can be estimated by summing all the above individual costs at every stage of the planning period as being expressed in the following equation:

$$
\mathrm{SC}_{T}=\mathrm{FC}_{T}+\mathrm{VC}_{T}
$$

\subsection{Outages Cost}

In power system cost-benefit analysis, the outages cost (OC) form a major part in the total system cost These costs are associated with that energy demanded but cannot be served by the system due to severe outages, and is known as the expected energy not served, ( $\epsilon$ ENS) . Outages cost is usually borne by the consumers as well as by the utility. The utility outages cost include loss of revenue, loss of goodwill, loss of future sales and increased maintenance and repair expenditure. However, the utility losses are seen to be insignificant compared with the losses incurred by its consumers when power interruptions and energy cease occur. The consumers perceive power 
outages and energy shortages differently. A residential consumer may suffer a great deal of anxiety and inconvenience if an outage occurs during a hot summer day or deprives him from domestic activities or causes food spoilage. For a commercial user, he will also suffer a great hardship and losses of being forced to close until power is restored. Also, an outage may cause a great damage to an industrial customer if it occurs disrupting and disabling the production processes [9] [10].

One method of evaluating the $\epsilon$ ENS is described in [11]. Therefore, for estimating the outages cost (OC) is to multiply the value of that $\epsilon$ ENS by an appropriate Outage Cost Rate (OCR), as follows:

$$
\mathrm{OC}_{T}=\sum_{t}(\epsilon \mathrm{ENS} \cdot \mathrm{OCR})^{t}
$$

where OCR: US\$/kwh and $\epsilon$ ENS : kWh lost.

The total cost of supplying the electric energy to the consumers is the sum of system cost that will generally increase as consumers are provided with higher reliability and customer outages cost that will, however, decrease as system reliability increases or vice versa. This total system cost (TSC) can be expressed as in the following equation:

$$
\mathrm{TSC}_{T}=\mathrm{SC}_{T}+\mathrm{OC}_{T}
$$

The prominent role of outage cost estimation, as revealed in the above equation, is to assess the worth of power system reliability by comparing this cost (OC) with the size of system investment (SC) in order to arrive at the least overall system cost that will establish the most appropriate system reliability level that ensures energy continuous flow as well as the least cost of its production.

The incorporation of customer outage costs in investment models for power system expansion plans is very difficult for planners in fast developing countries. This difficulty stems principally either from the lack of system records of outage data, failure rate, frequency, duration of repair etc., or the failure to carry out customer surveys to estimate the impact and severity of such outages in terms of monetary value.

\section{Models Developed for the Reliability and Cost Evaluation Utilized in This Study}

To perform the assessments and analyses of this study, a computer program containing four basic models has been developed at the King Saud University. These models, shown in Figure 2, assess the requirements of developing power systems in order to satisfy specified reliability and economic criteria and they are briefly described as follows:

SYSDAT model: This model prepares two essential data files, namely, the capacity file and the load file. The first one is known as the Capacity Outage Probability Table (COPT) which contains all the outage capacity states with their associated probabilities of occurrence. The second one is known as the Load Duration Curve (LDC) which arranges the load levels in a typical load variation curve in a descending order of magnitude starting with the maximum load and ending with the minimum load. This preparation process starts by the SYSDA at the beginning of each year of the planning period and then is sent to the SYSREL in the next stage of the planning process in order to perform system reliability evaluation task.

SYSREL model: Receives the data files of both the COPT and the LDC. These two files are then convolved (combined together) to yield the system evaluated risk level, i.e., the Loss of Load Expectation (LOLEe). This LOLEe is then compared with the risk level prescribed by the utility management (LOLEp). If The LOLEe exceeds the LOLEp, an additional capacity should be added to the system in order to maintain itsrisk level within the satisfactory and accepted level prescribed by management decision, otherwise it proceeds to the next year.

SYSENR model: estimates the energy served (ES) by each generating unit residing in the system as well as the energy not served (ENS) due to forced power outages. It adopts a priority loading order, i.e. the generating units are loaded according to their least operation cost. Hence, operating, first, the most efficient and economic operating units (called the base units), followed by the more costly operating units (called the intermediate units), then followed by the most costly operating units (called the peak units), and so on. This means that the least cost operating units occupy the lower levels in the LDC area, and the more expensive operating units occupy the upper levels in the LDC respectively.

SYSCOS model: computes all system pertinent costs mentioned in the scope and contained in the context of this study like: fixed costs (FC), variable cost (VC), outages cost (OC) and total system cost (TSC).

SYSCON model: evaluates system reliability levels (LOLE) for systems after being interconnected. It reveals 


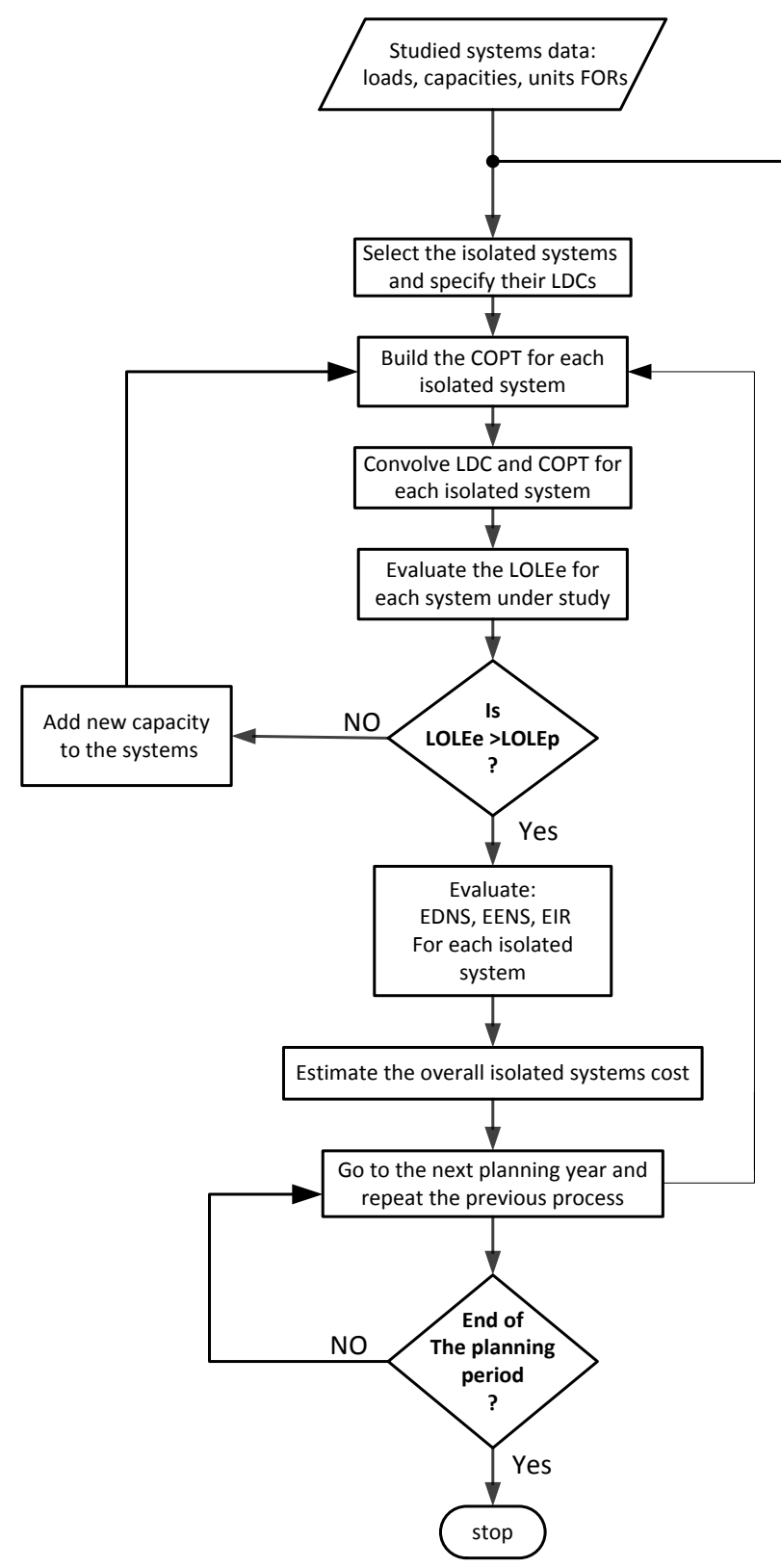

(a)

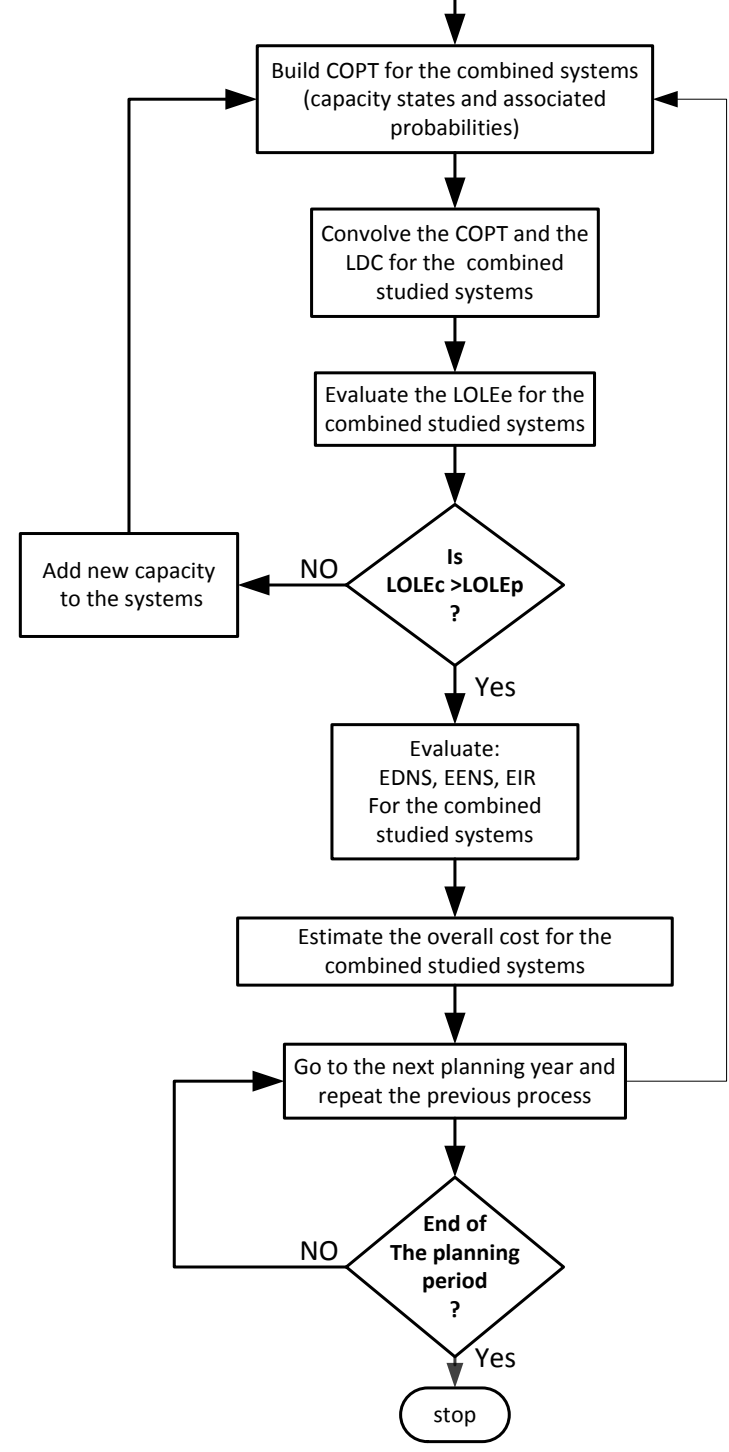

(b)

Figure 2. Flowchart for the proposed planning approach. (a) Isolated systems. (b) Interconnected systems.

the merits and advantages of system interconnection in terms of reliability level improvement and reserve capacity saving.

\section{Case Study}

The previous techniques have been applied to a particular case in a developing country. This case study is based on two real power systems situated in the southern part of the Kingdom of Saudi Arabia where are abbreviated in this study as systems A and B respectively. These two power systems are supposed to serve a major populated community with a potential future commercial and industrial load growth. The study considers that uncertainty is a vital aspect of power systems planning in developing countries that must be taken into consideration. Thus, the analysis procedure generally involves identifying the potential uncertain events and assigning a probability 
to the event occurrence. The impacts may then be probability-weighted, and a composite system impact value can be computed. This process may be repeated by examining alternative or contingency plans [12] [13].

\subsection{Separate and Integrated Systems}

Most power systems have grid interconnections either within the country or among neighboring countries. One objective reported in this paper is to evaluate the reliability benefits associated with the interconnection of power systems. Therefore, study is focused on reliability evaluation of systems A and B both as isolated systems and as interconnected systems. Analysis of this type enables the benefits, if any, that may accrue from integrated rather than isolated systems, to be explored as well as deciding viable generation expansion plans. Therefore, a 6-year expansion plan for systems A and B, assuming a reliability criterion (LOLE) of 0.1 days/year (frequently quoted as a practical value), is determined by implementing the methodology exhibited in Figure 2 . The analysis represents the expansion plans for both systems as being isolated and interconnected. A summary of these expansion plans is shown in Table 1 and plotted in Figure 3.

The two systems are reinforced whenever the reliability index exceeds the prescribed risk level at any year of the planning horizon. At years 2 and 4, when both systems reliability levels exceed the prescribed limit, unit (s) must be added. The results, displayed in Table 2, show that the number of units and the present value costs are reduced if the two systems are interconnected rather than being isolated which means saving in both units numbers and installation costs.

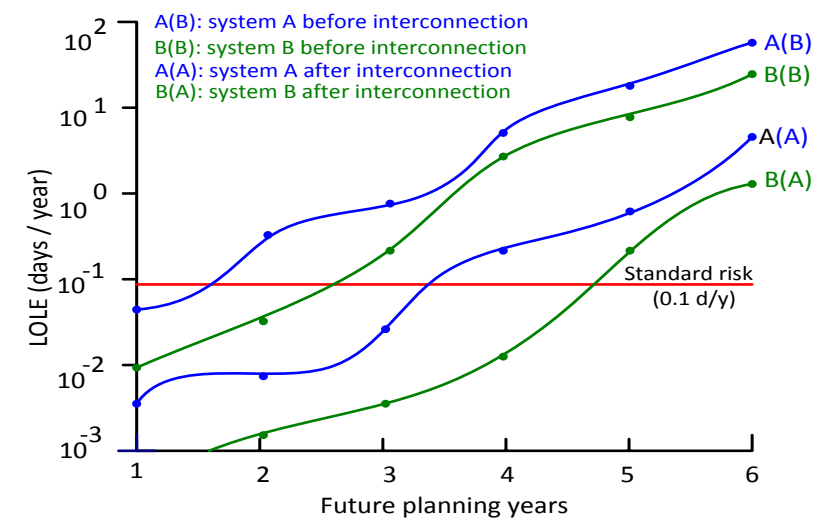

Figure 3. Variations of LOLE before and after interconnection.

Table 1. LOLE index for both systems as isolated and as interconnected.

\begin{tabular}{ccccc}
\hline Year & $\mathrm{A}(\mathrm{B})$ & $\mathrm{A}(\mathrm{A})$ & $\mathrm{B}(\mathrm{B})$ & $\mathrm{B}(\mathrm{A})$ \\
\hline 1 & 00.07234 & 0.00637 & 0.00926 & 0.00006 \\
2 & 00.68861 & 0.00709 & 0.06790 & 0.00313 \\
3 & 00.96242 & 0.08670 & 0.51488 & 0.00645 \\
4 & 00.85371 & 0.13842 & 4.62191 & 0.08838 \\
5 & 30.16790 & 0.75614 & 9.95545 & 0.21623 \\
6 & 80.93185 & 8.29678 & 30.60693 & 0.94383 \\
\hline
\end{tabular}

Table 2. Systems costs for isolated and interconnected systems.

\begin{tabular}{ccccccc}
\hline \multirow{2}{*}{ System } & \multicolumn{3}{c}{ Isolated } & \multicolumn{3}{c}{ Interconnected } \\
\cline { 2 - 7 } & No. of units & Cost (MUS\$) & $\epsilon$ ENS $($ MWh) & No. of units & Cost (MUS\$) & $\epsilon$ ENS (MWh) \\
\hline A & 3 & 18.62 & 8.652 & 2 & 9.44 & 1.054 \\
B & 2 & 10.42 & 6.852 & 1 & 6.75 & 2.045 \\
\hline
\end{tabular}


From the above analysis, it can be concluded that both systems will benefit from the interconnection in terms of saving in installation costs as well as reduction in size of energy interruptions. However, the next step must assess the economic and technical merits that may result from either building costly high voltage transmission lines in order to integrate isolated systems or instead, adding generating capacity to each system independently.

\subsection{Uncertainty in Future Loads Growth}

In some existing generation expansion planning methods it has been assumed that the forecast peak demands do not change. In fast developing countries, this assumption does not hold rigorously and there is always some degree of uncertainty in future loads growth forecasting. This uncertainty is likely to affect the system reliability levels and consequently to influence the capacity planning decision [8]. To investigate the uncertainty impact System "A" was chosen to be analyzed. The forecasted peak load was represented by a normal distribution having a standard deviation of $10 \%$ and this remained constant for the planning period. There are several important aspects associated with load uncertainty were evaluated such as system cost and outages costs using a load model having 7 discrete intervals. The effect of load uncertainty upon both system costs and outages costs are tested and the results are shown in Table 3 and depicted in Figure $\mathbf{4}$ which reveals that these costs (i.e. SC and OC) increase with increasing loads which implies reduction in the prescribed reliability level and hence requires more investment and operation costs [14].

\subsection{Uncertainty in Unit Installation Date}

In developing countries, a delay in unit installation date, due to probable undesirable economic conditions (e.g. lack of investment, rare resources, political havoc etc.) should be expected and taken into consideration in the planning horizon. Hence, to show the effect of more than on year's delay in unit installation dates has been investigated using system A. Figure 5 shows the effect of delaying unit installation date for an extended number of future years and its consequences upon both system cost (SC) and outage cost (OC). It is evident from the table that installation delay has an adverse impact upon system reliability index (LOLE) as well as system costs that are directly related to it.

If more uncertainties in installation dates are assumed, results depicted by Figure $\mathbf{4}$ show that, as unit deferring is increased, the outages cost increase rapidly but that the system cost steadily decreases. On the contrary, the timely installation has less effect on the outage costs than in the delayed case. Consequently, incentives should exist to justify decisions upon delaying or complying with the scheduled date of unit addition. One reason could be that it would be a catastrophic if unit installation is postponed for longer periods as shown in Figure 5.

In developing countries data collection is not an easy task and it is often difficult to establish probabilistic data for a system which did not have regular and organized collection of data for the use in probabilistic techniques. It is, therefore, important to establish systematic data collections describing all behavior aspects of power system which can then be used in reliability and economic evaluation for future planning and studies which are critically needed for power system planning in developing countries.

The effect of advancing unit installation date has been analyzed again using the same system A. The sensitivity analysis results are is shown in Table 4 and depicted in Figure 5, The results indicate that advancing unit

Table 3. Data for load forecast uncertainty.

\begin{tabular}{cccccc}
\hline $\begin{array}{c}\text { No. of } \\
\text { St. Dev. }\end{array}$ & Load Uncertainty (\%) & $\begin{array}{c}\text { Load Level } \\
\text { (MW) }\end{array}$ & Probability & $\begin{array}{c}\text { System cost } \\
\text { (MUS\$) }\end{array}$ & $\begin{array}{c}\text { Outages Costs } \\
\text { (MUS\$) }\end{array}$ \\
\hline-3 & -15 & 29 & 0.006 & 1 & 0.1 \\
-2 & -10 & 32 & 0.061 & 4 & 0.2 \\
-1 & -5 & 36 & 0.242 & 6 & 0.3 \\
0 & 0 & 40 & 0.382 & 8 & 0.4 \\
1 & 5 & 44 & 0.242 & 13 & 0.8 \\
2 & 10 & 48 & 0.061 & 17 & 1.3 \\
3 & 15 & 53 & 0.006 & 25 & 2.2 \\
\hline
\end{tabular}


Table 4. System costs variation for timely and (delayed) installation dates.

\begin{tabular}{|c|c|c|c|c|c|c|}
\hline Year & $\begin{array}{l}\text { Peak Load } \\
\text { (MW) }\end{array}$ & $\begin{array}{c}\text { LOLE } \\
\text { (days/yea) }\end{array}$ & Unit added & $\begin{array}{c}\text { SC } \\
\text { (MUS\$) }\end{array}$ & $\begin{array}{c}\text { OC } \\
\text { (MUS\$) }\end{array}$ & $\begin{array}{c}\text { Percentage } \\
\text { Increase (decrease) }\end{array}$ \\
\hline 1 & 435 & $0.02(2.1)$ & $0(0)$ & $00(00)$ & $2.2(2.2)$ & $2.0(2.3)$ \\
\hline 2 & 511 & 0.08 (1.8) & $0(0)$ & $00(00)$ & $4.4(4.4)$ & $5.4(5.4)$ \\
\hline 3 & 571 & $0.03(2.3)$ & $1(0)$ & $44(00)$ & $3.2(6.8)$ & $7.2(7.8)$ \\
\hline 4 & 632 & 0.09 (2.7) & $0(1)$ & $00(38)$ & $5.3(4.3)$ & $10.3(10.4)$ \\
\hline 5 & 712 & $0.05(3.2)$ & $1(0)$ & $31(00)$ & $4.9(7.4)$ & $13.7(14.8)$ \\
\hline
\end{tabular}

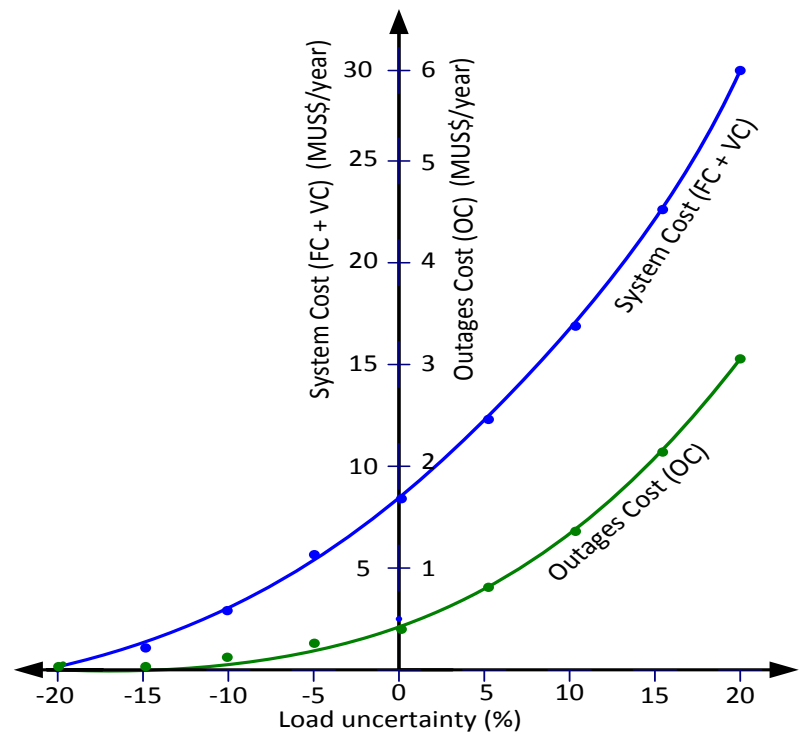

Figure 4. Effect of load uncertainty on system and outages costs.

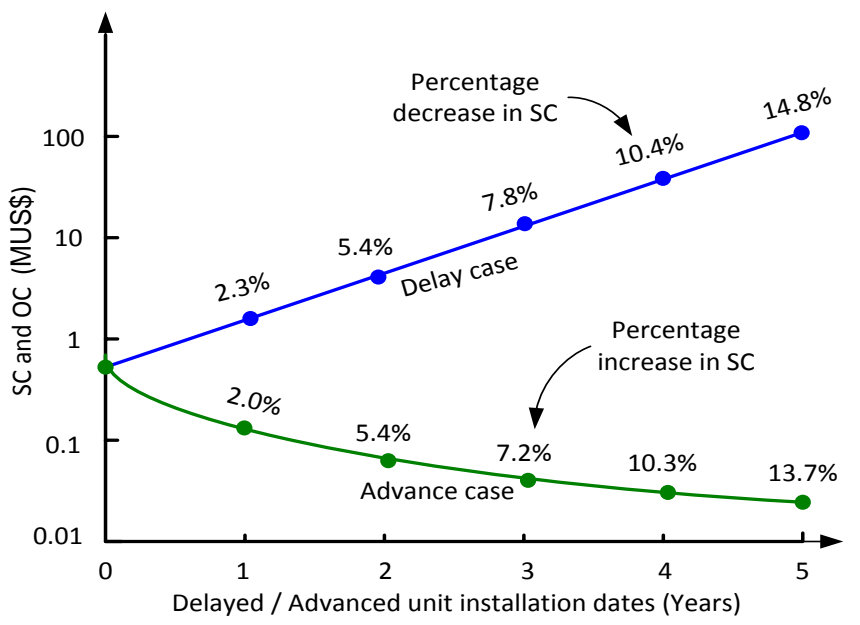

Figure 5. Effect of delay/advance in installation dates.

installation date has less effect compared with delay case on the outage cost In the advance case, outage costs are less sensitive because there is no critical need to advance the installation date. This can be argued on the basis that the system under analysis (System A) is reliable enough due to the selected reliability criterion of 0.1 days/year. However, the system cost is greater due to the earlier time investment. For convenient comparison, both delay case and advance case are plotted as function of successive future years. 


\section{Conclusions}

In this paper, significant issues that may arise in power system planning in developing countries have been considered, analyzed and discussed. Two major constraints associated with power planning process, namely, reliability and cost have been modeled and applied to particular systems expansion planning in a developing country. The results demonstrate the benefits and merits associated with both reliability and cost of interconnecting isolated power systems into an integrated system. The uncertainly in future loads growth and unit installation time can be costly and undesirable. Therefore, their effects should be anticipated and studied in order to mitigate their effects so that possible deterioration in system reliability level as well as unnecessary additional expenditure can be averted.

In developing countries data collection is not an easy task and it is often difficult to establish probabilistic data for a system which did not have regular and organized collection of data for the use in probabilistic techniques. It is, therefore, important to establish systematic data collections describing all behavior aspects of power system which can then be used in reliability and economic evaluation for future planning and studies which are critically needed for power system planning in developing countries.

\section{References}

[1] Covarrubias, A.J. (1988) Expert Systems and Power System Planning in Developing Countries. IEEE Conference Proceedings, Southeastcon'88, 11-13 April 1988, 551-555.

[2] Shaalan, A.M. (2011) Essential Aspects of Power System Planning in Developing Countries. Journal of King Saud University-Engineering Sciences, 23, 27-32.

[3] Schramm, G. (1990) Electric Power in Developing Countries: Status, Problems, Prospects. Annual Review of Power, 15, 307-333. http://dx.doi.org/10.1146/annurev.energy.15.1.307

[4] El-Zayyat, H., et al. (1991) Power System Planning in Developing Countries, the Case of Jordan. Mansoura Engineering Journal (MEJ), 16.

[5] Meier, P. (1990) Power Sector Innovation in Developing Countries: Implementing Investment Planning under Capital and Environmental Constraints. Annual Review of Power, 15, 277-306. http://dx.doi.org/10.1146/annurev.eg.15.110190.001425

[6] Agalgaonkar; A.P. and Kulkarni, S.V. (2006) Evaluation of Configuration Plans for DGs in Developing Countries Using Advanced Planning Techniques. IEEE Transaction on Power Systems, 21, 973-981.

[7] Wilbanks, T.J. (1990) Implementing Environmentally Sound Power Sector Strategies in Developing Countries. Annual Review of Power, 15, 255-276. http://dx.doi.org/10.1146/annurev.eg.15.110190.001351

[8] Sadeghzadeh, S.M. and Ansarian, M. (2006) Techno-Economic Power System Planning in Developing Countries. 7th IET International Conference on Advances in Power System Control, Operation and Management (APSCOM), Hong Kong, 30 October-2 November 2006, 216. http://dx.doi.org/10.1049/cp:20062083

[9] Billinton, R. and Allan, R.N. (1984) Reliability Evaluation of Power Systems. Pitman Books, London. http://dx.doi.org/10.1007/978-1-4615-7731-7

[10] Shaalan, A. M. (2012) Reliability Evaluation in Generation Expansion Planning Based on the Expected Energy Not Served. Journal of King Saud University (Engineering Sciences), 1.

[11] Shaalan, A.M. (1984) Reliability Evaluation in Long-Range Generation Expansion Planning. PhD Thesis, Victoria University of Manchester, Manchester, UK, April 1984, 51-58.

[12] Rudnick, H. and Quinteros, R. (1998) Power System Planning in the South America Electric Market Restructuring. VI Symposium of Specialists in Electric Operational and Expansion Planning —VI SEPOPE, Bahia, 24-29 May 1998.

[13] Weber, A., et al. (2013) Long-Term Power System Planning in the Context of Changing Policy Objectives. Workgroup for Infrastructure Policy (WIP), University of Technology, Berlin, June 2013.

[14] Flage, R. and Aven, T. (2009) On Treatment of Uncertainty in System Planning. Reliability Engineering \& System Safety, 94, 884-890. http://dx.doi.org/10.1016/j.ress.2008.09.011 